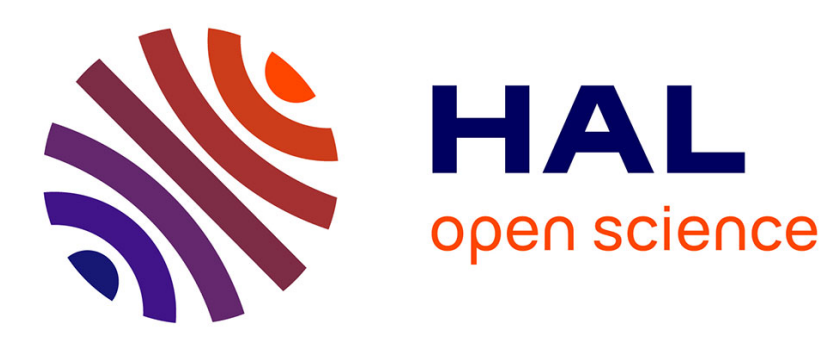

\title{
On the decay in time of solutions of the generalized regularized Boussinesq system
}

\author{
Youcef Mammeri
}

\section{To cite this version:}

Youcef Mammeri. On the decay in time of solutions of the generalized regularized Boussinesq system. Advances in Nonlinear Studies, 2010, 10, pp.837 - 849. hal-01090378

\section{HAL Id: hal-01090378 \\ https://hal.science/hal-01090378}

Submitted on 3 Dec 2014

HAL is a multi-disciplinary open access archive for the deposit and dissemination of scientific research documents, whether they are published or not. The documents may come from teaching and research institutions in France or abroad, or from public or private research centers.
L'archive ouverte pluridisciplinaire HAL, est destinée au dépôt et à la diffusion de documents scientifiques de niveau recherche, publiés ou non, émanant des établissements d'enseignement et de recherche français ou étrangers, des laboratoires publics ou privés. 


\title{
On the decay in time of solutions of the generalized regularized Boussinesq system
}

\author{
Y. Mammeri \\ Institut de mathématiques de Bordeaux, \\ CNRS UMR 5251, Université Bordeaux 1, \\ 33405 Talence Cedex, France. \\ Email: youcef.mammeri@math.u-bordeaux1.fr
}

November 2009

\begin{abstract}
We are interested in dispersive properties of the Boussinesq system for small initial data. We prove that, for a nonlinearity of sufficiently high order, the solution of the Boussinesq system exists for all time and tends to zero when the time tends to infinity.
\end{abstract}

Keywords. Boussinesq system, decay in time

MS Codes. 35B40, 35Q53, 76B03, 76B15

\section{Introduction}

The surface $\eta$ and the velocity $v$ of the two-way propagation of small amplitude, long wavelength, gravity waves in shallow water was first derived by Boussinesq $[5,11]$ from the Euler equation as a system of two equations of the form

$$
\begin{aligned}
\eta_{t}+v_{x}+(v \eta)_{x} & =0 \\
v_{t}+\eta_{x}+v v_{x}-v_{x x t} & =0
\end{aligned}
$$

The goal of this work is to study the decay in time for small amplitude solutions of a more general family of Boussinesq system (gBS) below [3, 4]

$$
\begin{aligned}
\eta_{t}+v_{x}+\left(v^{\rho} \eta\right)_{x}+\left(v \eta^{\rho}\right)_{x}+a v_{x x x}-b \eta_{x x t} & =0 \\
v_{t}+\eta_{x}+\left(v^{\rho+1}\right)_{x}+a \eta_{x x x}-b v_{x x t} & =0
\end{aligned}
$$

with $a \in \mathbb{R}, b>0$ and $\rho$ a positive integer.

For $s \in \mathbb{R}$, we denote $X^{s}(\mathbb{R})=H^{s}(\mathbb{R}) \cap L^{1}(\mathbb{R}) \times H^{s}(\mathbb{R}) \cap L^{1}(\mathbb{R})$ the space of functions $(\eta, v)$ such that the norm

$$
\|(\eta, v)\|_{X^{s}}:=\|\eta\|_{H^{s}}+\|v\|_{H^{s}}+\|\eta\|_{L^{1}}+\|v\|_{L^{1}}
$$

is finite. Our result reads as follows.

Theorem 1.1

Let $\rho \geq 4$. For $1 / 9 \leq \delta<1 / 3-2 /(3 \rho)$, we set $s=1 /(2 \delta)-1$. 
Then there exists $\varepsilon>0$ such that for all $\left(\eta_{0}, v_{0}\right) \in X^{s}(\mathbb{R})$ with $\left\|\left(\eta_{0}, v_{0}\right)\right\|_{X^{s}} \leq \varepsilon$, there exists a unique global in time solution $(\eta, v) \in \mathcal{C}\left(\mathbb{R} ; X^{s}(\mathbb{R})\right)$ of the $g B S$ system (1.1)-(1.2) with $\left(\eta_{0}, v_{0}\right)$ as initial datum. Moreover this solution verifies that there exists a constant $C>0$, depending only on $s$ and $\varepsilon$, such that for all time $t \in \mathbb{R}$, for all $x \in \mathbb{R}$, we have

$$
|\eta(x, t)|+|v(x, t)| \leq C(1+|t|)^{-\frac{1}{2}+\frac{3 \delta}{2}} .
$$

We notice that if $\delta$ tends to $1 / 9$ then the decay rate of the $L^{\infty}$-norm of $(\eta, v)(t)$ tends to $1 / 3$, but we need to impose more regularity on the initial datum. On the other hand, the more $\delta$ is close to $1 / 3$, the more the regularity is small, but the decay in time becomes slow.

We are inspired by the method described by Albert [1] for the generalized Benjamin-Bona-Mahony $(\mathrm{gBBM})$ equation $[2]$

$$
\eta_{t}+\eta_{x}+\eta^{\rho} \eta_{x}-\eta_{x x t}=0
$$

In this paper, Albert shows that, for a power $\rho>4$, the solution of (1.3), with a small initial datum, decreases in time with a rate of order $1 / 3$. A same result is found for the solution of the generalized Korteweg-de Vries equation [7, 10]

$$
\eta_{t}+\eta_{x}+\eta^{\rho} \eta_{x}+\eta_{x x x}=0 .
$$

In our work, we prove that the decay rate in time of the solution of the gBS system is also of order to $1 / 3$, but the result remains true for $\rho=4$. However, the gBBM equation can be considered as a special case of the gBS system. It is enough to take $v=\eta, a=0$ in the theorem 1.1 to slightly improve the result of Albert [1].

\section{Corollary 1.2}

Let $\rho \geq 4$. For $1 / 9 \leq \delta<1 / 3-2 /(3 \rho)$, we set $s=1 /(2 \delta)-1$.

Then there exists $\varepsilon>0$ such that for all $\eta_{0} \in H^{s}(\mathbb{R}) \cap L^{1}(\mathbb{R})$ with $\left\|\eta_{0}\right\|_{H^{s} \cap L^{1}} \leq \varepsilon$, there exists a unique global in time solution $\eta \in \mathcal{C}\left(\mathbb{R} ; H^{s}(\mathbb{R}) \cap L^{1}(\mathbb{R})\right)$ of the $g B B M$ equation (1.3) with $\eta_{0}$ as initial datum.

Moreover this solution verifies that there exists $C>0$, depending only on $s$ and $\varepsilon$, such that for all time $t \in \mathbb{R}$, for all $x \in \mathbb{R}$, we have

$$
|\eta(x, t)| \leq C(1+|t|)^{-\frac{1}{2}+\frac{3 \delta}{2}} .
$$

The Strauss method [10] will be used to prove these results. It consists of proving that, for a small initial datum, all the derivatives of the solution of the gBS system behave like a linear term, and so equivalent to a Sobolev norm of sufficiently high order, and the power $\rho$ relates to the dispersion. The decay in time is given by the free gBS system evolution.

We will use the following notations: for $1 \leq p<\infty$, we denote $L^{p}(\mathbb{R})$ the space of p-power integrable functions equipped with the norm

$$
\|f\|_{L^{p}}:=\left(\int_{-\infty}^{+\infty}|f(x)|^{p} d x\right)^{1 / p}
$$

we denote $L^{\infty}(\mathbb{R})$ the functions space equipped with the norm

$$
\|f\|_{\infty}=\sup \operatorname{ess}(f):=\inf \{c ;|f(x)| \leq c \text { almost everywhere in } \mathbb{R}\} .
$$

The Schwartz space is denoted by $\mathcal{S}(\mathbb{R})$ and for $1 \leq p \leq \infty, W^{s, p}(\mathbb{R})$ is the Sobolev space equipped with the norm

$$
\|f\|_{W^{s, p}}:=\left\|\left(1-\partial_{x}^{2}\right)^{s / 2} f\right\|_{L^{p}} .
$$

In particular, $H^{s}(\mathbb{R}):=W^{s, 2}(\mathbb{R})$. 
We organise the paper as follows. In the first section, we give estimates concerning the linear Cauchy problem. In the second one, the existence and the uniqueness of global solution with the decay in time are proved.

\section{Estimates for the linear gBS system}

We consider the linear Cauchy problem

$$
\begin{aligned}
\eta_{t}+v_{x}+a v_{x x x}-b \eta_{x x t} & =0 \\
v_{t}+\eta_{x}+a \eta_{x x x}-b v_{x x t} & =0 \\
\eta(x, 0)=\eta_{0}(x), v(x, 0) & =v_{0}(x),
\end{aligned}
$$

with $a \in \mathbb{R}, b>0$.

Let us suppose that the initial datum $\left(\eta_{0}, v_{0}\right)$ is in the Schwartz space $\mathcal{S}(\mathbb{R}) \times \mathcal{S}(\mathbb{R})$. The solution $(\eta, v)$ of the ordinary differential equation, deduced from the Fourier transform in space, is given by, for all $x \in \mathbb{R}$ and $t \in \mathbb{R}^{*}$,

$$
\begin{aligned}
\eta(x, t) & =\frac{1}{2 \pi} \int_{-\infty}^{+\infty} \mathrm{e}^{i k x} \cos \left(t k \frac{1-a k^{2}}{1+b k^{2}}\right) \hat{\eta}_{0}(k)-i \mathrm{e}^{i k x} \sin \left(t k \frac{1-a k^{2}}{1+b k^{2}}\right) \hat{v}_{0}(k) d k \\
& =\frac{1}{4 \pi} \int_{-\infty}^{+\infty}\left(\mathrm{e}^{i t h_{\alpha}(k)}+\mathrm{e}^{i t g_{\alpha}(k)}\right) \hat{\eta}_{0}(k)-\left(\mathrm{e}^{i t h_{\alpha}(k)}-\mathrm{e}^{i t g_{\alpha}(k)}\right) \hat{v}_{0}(k) d k \\
v(x, t) & =\frac{1}{2 \pi} \int_{-\infty}^{+\infty} \mathrm{e}^{i k x} \cos \left(t k \frac{1-a k^{2}}{1+b k^{2}}\right) \hat{v}_{0}(k)-i \mathrm{e}^{i k x} \sin \left(t k \frac{1-a k^{2}}{1+b k^{2}}\right) \hat{\eta}_{0}(k) d k \\
& =\frac{1}{4 \pi} \int_{-\infty}^{+\infty}\left(\mathrm{e}^{i t h_{\alpha}(k)}+\mathrm{e}^{i t g_{\alpha}(k)}\right) \hat{v}_{0}(k)-\left(\mathrm{e}^{i t h_{\alpha}(k)}-\mathrm{e}^{i t g_{\alpha}(k)}\right) \hat{\eta}_{0}(k) d k,
\end{aligned}
$$

with $h_{\alpha}(k)=k\left(1-a k^{2}\right) /\left(1+b k^{2}\right)+\alpha k, g_{\alpha}(k)=-k\left(1-a k^{2}\right) /\left(1+b k^{2}\right)+\alpha k$, and $\alpha=x / t$. We first recall the Van der Corput lemma [9].

\section{Lemma 2.1}

Let $n \geq 2$. Then there exists $C>0$ such that for all $\alpha \leq \beta, \lambda>0, t \in \mathbb{R}^{*}$ and for all function $h \in \mathcal{C}^{\infty}([\alpha, \beta])$ real valued satisfying for all $k \in[\alpha, \beta],\left|h^{(n)}(k)\right| \geq \lambda$, we have

$$
\left|\int_{a}^{b} e^{i t h(k)} d k\right| \leq \frac{C}{(\lambda|t|)^{1 / n}}
$$

The Van der Corput lemma is now applied with the functions $h_{\alpha}$ and $g_{\alpha}$.

\section{Lemma 2.2}

There exists $C>0$ such that for all $1 / 9 \leq \delta<1 / 3, \alpha \in \mathbb{R}$ and all time $|t|>((3+2 \sqrt{2}) / b)^{1 / 2 \delta}$, we have

$$
\left|\int_{-|t|^{\delta}}^{|t|^{\delta}} \mathrm{e}^{i t h_{\alpha}(k)} d k\right| \leq C|t|^{-\frac{1}{2}+\frac{3 \delta}{2}},\left|\int_{-|t|^{\delta}}^{|t|^{\delta}} \mathrm{e}^{i t g_{\alpha}(k)} d k\right| \leq C|t|^{-\frac{1}{2}+\frac{3 \delta}{2}} .
$$

Proof. We only consider the cases $h_{\alpha}$ and $k \geq 0$, the other cases being dealt with similarly. We have for $k \in\left[0,|t|^{\delta}\right]$

$$
\left|h_{\alpha}^{\prime \prime}(k)\right|=\left|\frac{2(a+b) k\left(b k^{2}-3\right)}{\left(1+b k^{2}\right)^{3}}\right|,\left|h_{\alpha}^{\prime \prime \prime}(k)\right|=\left|\frac{6(a+b)\left(b^{2} k^{4}-6 b k^{2}+1\right)}{\left(1+b k^{2}\right)^{4}}\right| .
$$


We notice that $\left|h_{\alpha}^{\prime \prime}(k)\right|$ vanishes if $k=0, \sqrt{3 / b}$ and $\left|h_{\alpha}^{\prime \prime \prime}(k)\right|$ vanishes if $k=\sqrt{(3-2 \sqrt{2}) / b}, \sqrt{(3+2 \sqrt{2}) / b}$. The interval $\left[0,|t|^{\delta}\right]$ is divided in four pieces $[0, A] \cup[A, B] \cup[B, C] \cup\left[C,|t|^{\delta}\right]$, with

$$
0<A<\sqrt{\frac{3-2 \sqrt{2}}{b}}<B<\sqrt{\frac{3}{b}}<C<\sqrt{\frac{3+2 \sqrt{2}}{b}}<|t|^{\delta} .
$$

In particular, for $k \in[0, A] \cup[B, C]$, we have $\left|h_{\alpha}^{\prime \prime \prime}(k)\right| \geq C>0$, for $k \in[A, B],\left|h_{\alpha}^{\prime \prime}(k)\right| \geq C>0$ and for $k \in\left[C,|t|^{\delta}\right],\left|h_{\alpha}^{\prime \prime}(k)\right| \geq\left|h_{\alpha}^{\prime \prime}\left(|t|^{\delta}\right)\right| \geq C|t|^{-3 \delta}$, where $C$ is independent on $t$. Finally, the lemma 2.1 is applied on each interval to give

$$
\left|\int_{0}^{|t|^{\delta}} \mathrm{e}^{i t h_{\alpha}(k)} d k\right| \leq C|t|^{-\frac{1}{3}}+C|t|^{-\frac{1}{2}+\frac{3 \delta}{2}}
$$

and since $\delta \geq 1 / 9$, we have $-1 / 2+3 \delta / 2 \geq-1 / 3$.

The oscillating integrals (2.1) and (2.2) can be majorized. When there is no ambiguity, we will write $C$ and $C_{s}$ the different constants appearing in the following results.

\section{Lemma 2.3}

Let $1 / 9 \leq \delta<1 / 3$ and $s=1 /(2 \delta)-1$. For all $f \in H^{s}(\mathbb{R}), \alpha \in \mathbb{R}$ and all time $|t|>((3+2 \sqrt{2}) / b)^{1 / 2 \delta}$, we have

$$
\left|\int_{|k|>|t|^{\delta}} \mathrm{e}^{i t h_{\alpha}(k)} \hat{f}(k) d k\right| \leq C_{s}|| f\left\|_{H^{s}}|t|^{-\frac{1}{2}+\frac{3 \delta}{2}},\left|\int_{|k|>|t|^{\delta}} \mathrm{e}^{i t g_{\alpha}(k)} \hat{f}(k) d k\right| \leq C_{s}|| f\right\|_{H^{s}}|t|^{-\frac{1}{2}+\frac{3 \delta}{2}} .
$$

Proof. We have

$$
\left|\int_{|t|^{\delta}}^{+\infty} \mathrm{e}^{i t h_{\alpha}(k)} \hat{f}(k) d k\right| \leq \int_{|t|^{\delta}}^{+\infty}|\hat{f}(k)| d k=\int_{|t|^{\delta}}^{+\infty} \frac{(1+k)^{s}}{(1+k)^{s}}|\hat{f}(k)| d k
$$

and the Cauchy-Schwarz inequality gives

$$
\left|\int_{|t|^{\delta}}^{+\infty} \mathrm{e}^{i t h_{\alpha}(k)} \hat{f}(k) d k\right| \leq\left(\int_{|t|^{\delta}}^{+\infty}(1+k)^{2 s}|\hat{f}(k)|^{2} d k\right)^{1 / 2}\left(\int_{|t|^{\delta}}^{+\infty} \frac{d k}{(1+k)^{2 s}}\right)^{1 / 2} .
$$

On one hand, since $s>1 / 2$, it gets

$$
\begin{aligned}
\left(\int_{|t|^{\delta}}^{+\infty} \frac{d k}{(1+k)^{2 s}}\right)^{1 / 2} & =\left(\left[\frac{-1}{(2 s-1)(1+k)^{2 s-1}}\right]_{|t|^{\delta}}^{+\infty}\right)^{1 / 2} \\
& \leq C_{s}|t|^{\frac{\delta}{2}(1-2 s)}=C_{s}|t|^{-\frac{1}{2}+\frac{3 \delta}{2}}
\end{aligned}
$$

On the other hand, since for $k \in \mathbb{R}$, we have $(1+k)^{2} \leq 2\left(1+k^{2}\right)$, the first integral becomes

$$
\begin{aligned}
\left(\int_{|t|^{\delta}}^{+\infty}(1+k)^{2 s}|\hat{f}(k)|^{2} d k\right)^{1 / 2} & \leq \sqrt{2^{s}}\left(\int_{|t|^{\delta}}^{+\infty}\left(1+k^{2}\right)^{s}|\hat{f}(k)|^{2} d k\right)^{1 / 2} \\
& \leq C_{s}|| f \|_{H^{s}}
\end{aligned}
$$

Finally, we find

$$
\left|\int_{|t|^{\delta}}^{+\infty} \mathrm{e}^{i t h_{\alpha}(k)} \hat{f}(k) d k\right| \leq C_{s}\|f\|_{H^{s}}|t|^{-\frac{1}{2}+\frac{3 \delta}{2}}
$$




\section{Proposition 2.4}

Let $1 / 9 \leq \delta<1 / 3$ and $s=1 /(2 \delta)-1$. There exists a constant $C_{s}>0$, depending only on $s$, such that for all function $f \in L^{1}(\mathbb{R}) \cap H^{s}(\mathbb{R}), \alpha \in \mathbb{R}$ and all time $t \in \mathbb{R}$, we have

$$
\begin{aligned}
\left|\int_{-\infty}^{+\infty} \mathrm{e}^{i t h_{\alpha}(k)} \hat{f}(k) d k\right| \leq C_{s}\left(\|f\|_{L^{1}}+\|f\|_{H^{s}}\right)(1+|t|)^{-\frac{1}{2}+\frac{3 \delta}{2}} \\
\left|\int_{-\infty}^{+\infty} \mathrm{e}^{i t g_{\alpha}(k)} \hat{f}(k) d k\right| \leq C_{s}\left(\|f\|_{L^{1}}+\|f\|_{H^{s}}\right)(1+|t|)^{-\frac{1}{2}+\frac{3 \delta}{2}} .
\end{aligned}
$$

Proof. Let $1 / 9 \leq \delta<1 / 3$ and $|t|>((3+2 \sqrt{2}) / b)^{1 / 2 \delta}=: k_{1}$, we write

$$
\int_{0}^{+\infty} \mathrm{e}^{i t h_{\alpha}(k)} \hat{f}(k) d k=\int_{0}^{|t|^{\delta}} \mathrm{e}^{i t h_{\alpha}(k)} \hat{f}(k) d k+\int_{|t|^{\delta}}^{+\infty} \mathrm{e}^{i t h_{\alpha}(k)} \hat{f}(k) d k .
$$

For the first integral, the Fubini theorem implies

$$
\begin{aligned}
\int_{0}^{|t|^{\delta}} \mathrm{e}^{i t h_{\alpha}(k)} \hat{f}(k) d k & =\int_{0}^{|t|^{\delta}} \mathrm{e}^{i t h_{\alpha}(k)}\left(\int_{-\infty}^{+\infty} \mathrm{e}^{-i k x^{\prime}} f\left(x^{\prime}\right) d x^{\prime}\right) d k \\
& =\int_{-\infty}^{+\infty}\left(\int_{0}^{|t|^{\delta}} \mathrm{e}^{i k\left(x-x^{\prime}\right)} \mathrm{e}^{-i t \frac{k\left(1-a k^{2}\right)}{1+b k^{2}}} d k\right) f\left(x^{\prime}\right) d x^{\prime} .
\end{aligned}
$$

We deduce that

$$
\left|\int_{0}^{|t|^{\delta}} \mathrm{e}^{i t h_{\alpha}(k)} \hat{f}(k) d k\right| \leq\left\|\int_{0}^{|t|^{\delta}} \mathrm{e}^{i t h_{\alpha}(k)} d k\right\|\left\|_{\infty}\right\| f \|_{L^{1}} .
$$

The lemmas 2.2 and 2.3 provide

$$
\left|\int_{0}^{+\infty} \mathrm{e}^{i t h_{\alpha}(k)} \hat{f}(k) d k\right| \leq C_{s}\left(\|f\|_{L^{1}}+\|f\|_{H^{s}}\right)|t|^{-\frac{1}{2}+\frac{3 \delta}{2}} .
$$

Since $|t|>k_{1}$, we have $1+|t|<\left(1 / k_{1}+1\right)|t|$, thus we obtain

$$
\left|\int_{0}^{+\infty} \mathrm{e}^{i t h_{\alpha}(k)} \hat{f}(k) d k\right| \leq C_{s}\left(\|f\|_{L^{1}}+\|f\|_{H^{s}}\right)(1+|t|)^{-\frac{1}{2}+\frac{3 \delta}{2}} .
$$

The contribution for $k \leq 0$ is dealt with similarly.

Let $|t| \leq k_{1}$. We immediately have

$$
\left|\int_{-\infty}^{+\infty} \mathrm{e}^{i t h_{\alpha}(k)} \hat{f}(k) d k\right| \leq \int_{-\infty}^{+\infty}|\hat{f}(k)| d k=\int_{-\infty}^{+\infty} \frac{\left(1+k^{2}\right)^{s / 2}}{\left(1+k^{2}\right)^{s / 2}}|\hat{f}(k)| d k,
$$

and the Cauchy-Schwarz inequality gives, with $s>1 / 2$,

$$
\left|\int_{-\infty}^{+\infty} \mathrm{e}^{i t h_{\alpha}(k)} \hat{f}(k) d k\right| \leq C_{s}\|f\|_{H^{s}}
$$

Since $|t| \leq k_{1}$, we have $(1+|t|) /\left(1+k_{1}\right) \leq 1$, thus

$$
\left|\int_{-\infty}^{+\infty} \mathrm{e}^{i t h_{\alpha}(k)} \hat{f}(k) d k\right| \leq C_{s}\|f\|_{H^{s}}(1+|t|)^{-\frac{1}{2}+\frac{3 \delta}{2}} .
$$

The result is obtained from the inequalities (2.3) and (2.4).

We can generalize the preceding result. 


\section{Corollary 2.5}

Let $1 / 9 \leq \delta<1 / 3$ and $s=1 /(2 \delta)-1$. There exists a constant $C_{s}>0$, depending only on $s$, such that for all function $f \in L^{p}(\mathbb{R}) \cap H^{s}(\mathbb{R})$, with $1 \leq p \leq 2$, for all $\alpha \in \mathbb{R}$ and all time $t \in \mathbb{R}$, we have

$$
\begin{aligned}
& \left\|\int_{-\infty}^{+\infty} \mathrm{e}^{i t g_{\alpha}(k)} \hat{f}(k) d k\right\|_{L^{q}} \leq C_{s}\left(\|f\|_{L^{p}}+\|f\|_{H^{s}}\right)(1+|t|)^{\left(-\frac{1}{2}+\frac{3 \delta}{2}\right)\left(1-\frac{2}{q}\right)} \\
& \left\|\int_{-\infty}^{+\infty} \mathrm{e}^{i t g_{\alpha}(k)} \hat{f}(k) d k\right\|_{L^{q}} \leq C_{s}\left(\|f\|_{L^{p}}+\|f\|_{H^{s}}\right)(1+|t|)^{\left(-\frac{1}{2}+\frac{3 \delta}{2}\right)\left(1-\frac{2}{q}\right)}
\end{aligned}
$$

where $1 / p+1 / q=1$.

Proof. See [8] for a complete proof by interpolation.

We apply the proposition 2.4 to the linear Cauchy problem.

\section{Corollary 2.6}

Let $1 / 9 \leq \delta<1 / 3$ and $s=1 /(2 \delta)-1$. There exists $C_{s}>0$, depending only on $s$, such that for all function $\left(\eta_{0}, v_{0}\right) \in\left(L^{1}(\mathbb{R}) \cap H^{s}(\mathbb{R})\right)^{2}, \alpha \in \mathbb{R}$ and all time $t \in \mathbb{R}$, we have

$$
\begin{aligned}
\left|\frac{1}{4 \pi} \int_{-\infty}^{+\infty}\left(\mathrm{e}^{i t h_{\alpha}(k)}+\mathrm{e}^{i t g_{\alpha}(k)}\right) \hat{\eta}_{0}(k)-\left(\mathrm{e}^{i t h_{\alpha}(k)}-\mathrm{e}^{i t g_{\alpha}(k)}\right) \hat{v}_{0}(k) d k\right| \\
\leq C_{s}\left(\left\|\eta_{0}\right\|_{L^{1}}+\left\|v_{0}\right\|_{L^{1}}+\left\|\eta_{0}\right\|_{H^{s}}+\left\|v_{0}\right\|_{H^{s}}\right)(1+|t|)^{-\frac{1}{2}+\frac{3 \delta}{2}} \\
\left|\frac{1}{4 \pi} \int_{-\infty}^{+\infty}\left(\mathrm{e}^{i t h_{\alpha}(k)}+\mathrm{e}^{i t g_{\alpha}(k)}\right) \hat{v}_{0}(k)-\left(\mathrm{e}^{i t h_{\alpha}(k)}-\mathrm{e}^{i t g_{\alpha}(k)}\right) \hat{\eta}_{0}(k) d k\right| \\
\leq C_{s}\left(\left\|\eta_{0}\right\|_{L^{1}}+\left\|v_{0}\right\|_{L^{1}}+\left\|\eta_{0}\right\|_{H^{s}}+\left\|v_{0}\right\|_{H^{s}}\right)(1+|t|)^{-\frac{1}{2}+\frac{3 \delta}{2}} .
\end{aligned}
$$

\section{Preliminary results}

We now quote some useful results. The fractional Leibniz rule will be used [6, lemma X.4].

\section{Theorem 3.1}

For all $s \geq 0$ and $1<p<+\infty$, there exists $C_{s, p}>0$, depending on $s$ and $p$ such that for all $u$ and $v$ in $\mathcal{S}(\mathbb{R})$, we have

$$
\|u v\|_{W^{s, p}} \leq C_{s, p}\left(\|u\|_{W^{s, p}}\|v\|_{\infty}+\|u\|_{\infty}\|v\|_{W^{s, p}}\right) .
$$

We will need an integration's lemma proved in [8].

\section{Lemma 3.2}

Let $\alpha>1$ and $0<\beta<1$. Then there exists $C>0$ such that for all time $t \geq 0$

$$
\int_{0}^{t} \frac{d \tau}{(1+\tau)^{\alpha}(1+t-\tau)^{\beta}} \leq \frac{C}{(1+t)^{\beta}} .
$$




\section{Existence and Uniqueness of global solution of the gBS system}

We consider the nonlinear Cauchy problem

$$
\begin{aligned}
\eta_{t}+v_{x}+\left(v^{\rho} \eta\right)_{x}+\left(v \eta^{\rho}\right)_{x}+a v_{x x x}-b \eta_{x x t} & =0 \\
v_{t}+\eta_{x}+\left(v^{\rho+1}\right)_{x}+a \eta_{x x x}-b v_{x x t} & =0 \\
\eta(x, 0)=\eta_{0}(x), v(x, 0) & =v_{0}(x),
\end{aligned}
$$

with $a \in \mathbb{R}, b>0$ and $\rho$ a positive integer. The main result of this paper is now proved.

Proof of the theorem 1.1. The proof is done for positive times.

To simplify the writings, we define the operator $\partial_{x} /\left(1-b \partial_{x}^{2}\right)$ by the Fourier symbol $\sigma(k):=i k /\left(1+b k^{2}\right)$, and we also denote $0<\theta:=(1 / 2-3 \delta / 2) \leq 1 / 3$.

Our assumptions on $\rho$ and $\delta$ imply that

$$
\theta \rho>1 .
$$

According to the Duhamel formula, $(\eta, v)$ is the solution of the gBS system (4.1)-(4.2)-(4.3) if and only if $(\eta, v)$ is the solution of the following equation, for $t \geq 0$

$$
\Phi(\eta, v)(t)=S_{t}\left(\eta_{0}, v_{0}\right)-\int_{0}^{t} S_{t-\tau}\left(\frac{\partial_{x}}{1-b \partial_{x}^{2}}\left(v^{\rho} \eta+v \eta^{\rho}, v^{\rho+1}\right)\right)(\tau) d \tau
$$

where $S_{t}(\eta, v)$ is defined by (2.1)-(2.2).

First of all, we notice that, for $f \in \mathcal{S}(\mathbb{R})$

$$
\left\|\frac{\partial_{x}}{1-b \partial_{x}^{2}} f\right\|_{H^{s}}=\left(\int_{-\infty}^{+\infty}\left(1+k^{2}\right)^{s}\left(\frac{i k}{1+b k^{2}}\right)^{2}|\widehat{f}(k)|^{2} d k\right)^{1 / 2} \leq\|f\|_{H^{s-1}} \leq\|f\|_{H^{s}} .
$$

On the other hand, we have

$$
\left\|\frac{\partial_{x}}{1-b \partial_{x}^{2}} f\right\|_{L^{1}}=\left\|\left(-\frac{1}{2 b^{3 / 2}} \operatorname{sgn}(x) \mathrm{e}^{-|x| / \sqrt{b}}\right) * f\right\|_{L^{1}} \leq C\|f\|_{L^{1}}
$$

Let $T>0$, we define the norm $N_{T}$ by, for $(\eta, v) \in X^{s}(\mathbb{R})$,

$$
N_{T}(\eta, v):=\sup _{0 \leq \tau \leq T}\left[\left(\|\eta\|_{L^{\infty}}(\tau)+\|v\|_{L^{\infty}}(\tau)\right)(1+\tau)^{\theta}+\|(\eta, v)\|_{X^{s}}(\tau)\right] .
$$

We separately prove some technical lemmas.

\section{Lemma 4.1}

There exists a constant $C_{s}>0$ such that for all $\left(\eta_{1}, v_{1}\right)$ and $\left(\eta_{2}, v_{2}\right)$ in $X^{s}(\mathbb{R})$, we have

$\left|\Phi\left(\eta_{1}, v_{1}\right)-\Phi\left(\eta_{2}, v_{2}\right)\right|(t) \leq C_{s}\left(N_{T}\left(\eta_{1}, v_{1}\right)^{\rho}+\sum_{i=0}^{\rho} N_{T}\left(\eta_{1}, v_{1}\right)^{\rho-i} N_{T}\left(\eta_{2}, v_{2}\right)^{i}\right) \times(1+t)^{-\theta} N_{T}\left(\eta_{1}-\eta_{2}, v_{1}-v_{2}\right)$.

Proof. Let $\left(\eta_{1}, v_{1}\right)$ and $\left(\eta_{2}, v_{2}\right)$ be in $X^{s}(\mathbb{R})$, the Duhamel formula gives

$$
\left|\Phi\left(\eta_{1}, v_{1}\right)-\Phi\left(\eta_{2}, v_{2}\right)\right|(t) \leq \int_{0}^{t}\left|S_{t-\tau}\left(\frac{\partial_{x}}{1-b \partial_{x}^{2}}\left(v_{1}^{\rho} \eta_{1}-v_{2}^{\rho} \eta_{2}+v_{1} \eta_{1}^{\rho}-v_{2} \eta_{2}^{\rho}, v_{1}^{\rho+1}-v_{2}^{\rho+1}\right)\right)\right|(\tau) d \tau
$$


The corollary 2.6 implies that there exists $C_{s}>0$, depending only on $s$, such that

$\left|\Phi\left(\eta_{1}, v_{1}\right)-\Phi\left(\eta_{2}, v_{2}\right)\right|(t) \leq C_{s} \int_{0}^{t} \frac{\left(\left\|\left(\frac{\partial_{x}}{1-b \partial_{x}^{2}}\left(v_{1}^{\rho} \eta_{1}-v_{2}^{\rho} \eta_{2}+v_{1} \eta_{1}^{\rho}-v_{2} \eta_{2}^{\rho}\right), \frac{\partial_{x}}{1-b \partial_{x}^{2}}\left(v_{1}^{\rho+1}-v_{2}^{\rho+1}\right)\right)\right\|_{X^{s}}\right)(\tau)}{(1+t-\tau)^{\theta}} d \tau$,

and the inequalities (4.6) and (4.7) provide

$$
\begin{aligned}
\left|\Phi\left(\eta_{1}, v_{1}\right)-\Phi\left(\eta_{2}, v_{2}\right)\right|(t) \leq & C_{s} \int_{0}^{t} \frac{\left(\left\|v_{1}^{\rho} \eta_{1}-v_{2}^{\rho} \eta_{2}+v_{1} \eta_{1}^{\rho}-v_{2} \eta_{2}^{\rho}\right\|_{L^{1}}+\left\|v_{1}^{\rho} \eta_{1}-v_{2}^{\rho} \eta_{2}+v_{1} \eta_{1}^{\rho}-v_{2} \eta_{2}^{\rho}\right\|_{H^{s}}\right)(\tau)}{(1+t-\tau)^{\theta}} d \tau \\
& +C_{s} \int_{0}^{t} \frac{\left(\left\|v_{1}^{\rho+1}-v_{2}^{\rho+1}\right\|_{L^{1}}+\left\|v_{1}^{\rho+1}-v_{2}^{\rho+1}\right\|_{H^{s}}\right)(\tau)}{(1+t-\tau)^{\theta}} d \tau
\end{aligned}
$$

From now, when there is no ambiguity, we use the following notation: for any positive $A$ and $B$, the notation $A \lesssim B$ means that there exists a constant $C>0$ such that $A \leq C B$.

Since $v_{1}^{\rho+1}-v_{2}^{\rho+1}=\left(v_{1}-v_{2}\right) \sum_{i=0}^{\rho} v_{1}^{\rho-i} v_{2}^{i}$, we have according to the fractional Leibniz theorem 3.1 and the Minkowski inequality

$$
\begin{aligned}
\left\|v_{1}^{\rho+1}-v_{2}^{\rho+1}\right\|_{H^{s}}(\tau) & \lesssim\left\|v_{1}-v_{2}\right\|_{H^{s}} \sum_{i=0}^{\rho}\left\|v_{1}^{\rho-i} v_{2}^{i}\right\|_{L^{\infty}}(\tau)+\left\|v_{1}-v_{2}\right\|_{L^{\infty}} \sum_{i=0}^{\rho}\left\|v_{1}^{\rho-i} v_{2}^{i}\right\|_{H^{s}}(\tau) \\
& =\mathrm{I}(\tau)+\mathrm{II}(\tau) .
\end{aligned}
$$

According to the definition (4.8) of the norm $N_{T}$, we can note that the decay in time relates to the $L^{\infty}$-norm. We deduce

$$
\begin{aligned}
\mathrm{I}(\tau) & \lesssim\left\|v_{1}-v_{2}\right\|_{H^{s}} \sum_{i=0}^{\rho}\left\|v_{1}\right\|_{L^{\infty}}^{\rho-i}\left\|v_{2}\right\|_{L^{\infty}}^{i}(\tau) \\
& \lesssim\left\|v_{1}-v_{2}\right\|_{H^{s}} \sum_{i=0}^{\rho}\left(\left\|v_{1}\right\|_{L^{\infty}} \frac{(1+\tau)^{\theta}}{(1+\tau)^{\theta}}\right)^{\rho-i}\left(\left\|v_{2}\right\|_{L^{\infty}} \frac{(1+\tau)^{\theta}}{(1+\tau)^{\theta}}\right)^{i} \\
& \lesssim\left(\sum_{i=0}^{\rho} N_{T}\left(\eta_{1}, v_{1}\right)^{\rho-i} N_{T}\left(\eta_{2}, v_{2}\right)^{i}\right)(1+\tau)^{-\theta \rho} N_{T}\left(\eta_{1}-\eta_{2}, v_{1}-v_{2}\right)
\end{aligned}
$$

For II $(\tau)$, the theorem 3.1 is applied again to find

$$
\begin{aligned}
\mathrm{II}(\tau) \lesssim\left\|v_{1}-v_{2}\right\|_{\infty} & \left(\left\|v_{2}\right\|_{\infty}^{\rho-1}\left\|v_{2}\right\|_{H^{s}}+\sum_{i=0}^{\rho-1}\left\|v_{1}\right\|_{\infty}^{\rho-i-1}\left\|v_{1}\right\|_{H^{s}}\left\|v_{2}\right\|_{\infty}^{i}\right. \\
& \left.+\left\|v_{1}\right\|_{\infty}^{\rho-1}\left\|v_{1}\right\|_{H^{s}}+\sum_{i=1}^{\rho}\left\|v_{1}\right\|_{\infty}^{\rho-i}\left\|v_{2}\right\|_{\infty}^{i-1}\left\|v_{2}\right\|_{H^{s}}\right)(\tau),
\end{aligned}
$$

and as for $\mathrm{I}(\tau)$, it gets

$$
\operatorname{II~}(\tau) \lesssim\left(\sum_{i=0}^{\rho} N_{T}\left(\eta_{1}, v_{1}\right)^{\rho-i} N_{T}\left(\eta_{2}, v_{2}\right)^{i}\right)(1+\tau)^{-\theta \rho} N_{T}\left(\eta_{1}-\eta_{2}, v_{1}-v_{2}\right) .
$$

We also have

$$
\left\|v_{1}^{\rho+1}-v_{2}^{\rho+1}\right\|_{L^{1}}(\tau) \leq\left\|v_{1}-v_{2}\right\|_{L^{1}} \sum_{i=0}^{\rho}\left\|v_{1}\right\|_{\infty}^{\rho-i}\left\|v_{2}\right\|_{\infty}^{i}(\tau)
$$




$$
\lesssim\left(\sum_{i=0}^{\rho} N_{T}\left(\eta_{1}, v_{1}\right)^{\rho-i} N_{T}\left(\eta_{2}, v_{2}\right)^{i}\right)(1+\tau)^{-\theta \rho} N_{T}\left(\eta_{1}-\eta_{2}, v_{1}-v_{2}\right)
$$

By noticing that $v_{1}^{\rho} \eta_{1}-v_{2}^{\rho} \eta_{2}=v_{1}^{\rho}\left(\eta_{1}-\eta_{2}\right)+\eta_{2}\left(v_{1}-v_{2}\right) \sum_{i=0}^{\rho-1} v_{1}^{\rho-1-i} v_{2}^{i}$, we obtain in the same manner

$$
\begin{aligned}
\left\|v_{1}^{\rho} \eta_{1}-v_{2}^{\rho} \eta_{2}\right\|_{H^{s}}(\tau) & \lesssim\left(N_{T}\left(\eta_{1}, v_{1}\right)^{\rho}+\sum_{i=1}^{\rho} N_{T}\left(\eta_{1}, v_{1}\right)^{\rho-i} N_{T}\left(\eta_{2}, v_{2}\right)^{i}\right) \times(1+\tau)^{-\theta \rho} N_{T}\left(\eta_{1}-\eta_{2}, v_{1}-v_{2}\right) \\
\left\|v_{1}^{\rho} \eta_{1}-v_{2}^{\rho} \eta_{2}\right\|_{L^{1}}(\tau) & \lesssim\left(N_{T}\left(\eta_{1}, v_{1}\right)^{\rho}+\sum_{i=1}^{\rho} N_{T}\left(\eta_{1}, v_{1}\right)^{\rho-i} N_{T}\left(\eta_{2}, v_{2}\right)^{i}\right) \times(1+\tau)^{-\theta \rho} N_{T}\left(\eta_{1}-\eta_{2}, v_{1}-v_{2}\right)
\end{aligned}
$$

and idem for $v_{1} \eta_{1}^{\rho}-v_{2} \eta_{2}^{\rho}$. Finally, it remains

$$
\left|\Phi\left(\eta_{1}, v_{1}\right)-\Phi\left(\eta_{1}, v_{1}\right)\right|(t) \lesssim\left(N_{T}\left(\eta_{1}, v_{1}\right)^{\rho}+\sum_{i=0}^{\rho} N_{T}\left(\eta_{1}, v_{1}\right)^{\rho-i} N_{T}\left(\eta_{2}, v_{2}\right)^{i}\right) \int_{0}^{t} \frac{d \tau}{(1+\tau)^{\theta \rho}(1+t-\tau)^{\theta}} N_{T}\left(\eta_{1}-\eta_{2}, v_{1}-v_{2}\right) .
$$

Since $\theta \rho>1$ and $0<\theta \leq 1 / 3<1$, we conclude thanks to the lemma 3.2 .

\section{Lemma 4.2}

There exists $C_{s}>0$ such that for all $\left(\eta_{1}, v_{1}\right)$ and $\left(\eta_{2}, v_{2}\right)$ in $X^{s}(\mathbb{R})$, we have

$$
\left\|\Phi\left(\eta_{1}, v_{1}\right)-\Phi\left(\eta_{2}, v_{2}\right)\right\|_{X^{s}}(t) \leq C_{s}\left(\left(N_{T}\left(\eta_{1}, v_{1}\right)^{\rho}+\sum_{i=0}^{\rho} N_{T}\left(\eta_{1}, v_{1}\right)^{\rho-i} N_{T}\left(\eta_{2}, v_{2}\right)^{i}\right) N_{T}\left(\eta_{1}-\eta_{2}, v_{1}-v_{2}\right) .\right.
$$

Proof. We now majorize the norm $\left\|\Phi\left(\eta_{1}, v_{1}\right)-\Phi\left(\eta_{2}, v_{2}\right)\right\|_{X^{s}}(t)$.

$$
\left\|\Phi\left(\eta_{1}, v_{1}\right)-\Phi\left(\eta_{2}, v_{2}\right)\right\|_{X^{s}}(t) \leq \int_{0}^{t}\left\|S_{t-\tau}\left(\frac{\partial_{x}}{1-b \partial_{x}^{2}}\left(v_{1}^{\rho} \eta_{1}-v_{2}^{\rho} \eta_{2}+v_{1} \eta_{1}^{\rho}-v_{2} \eta_{2}^{\rho}, v_{1}^{\rho+1}-v_{2}^{\rho+1}\right)\right)\right\|_{X^{s}}(\tau) d \tau
$$

the inequalities (4.6) and (4.7) imply

$$
\left\|\Phi\left(\eta_{1}, v_{1}\right)-\Phi\left(\eta_{2}, v_{2}\right)\right\|_{X^{s}}(t) \leq \int_{0}^{t}\left\|\left(v_{1}^{\rho} \eta_{1}-v_{2}^{\rho} \eta_{2}+v_{1} \eta_{1}^{\rho}-v_{2} \eta_{2}^{\rho}, v_{1}^{\rho+1}-v_{2}^{\rho+1}\right)\right\|_{X^{s}}(\tau) d \tau
$$

Since $X^{s}(\mathbb{R})=\left(H^{s}(\mathbb{R}) \cap L^{1}(\mathbb{R})\right)^{2}$, as for the preceding lemma, we obtain

$$
\begin{aligned}
\left\|\Phi\left(\eta_{1}, v_{1}\right)-\Phi\left(\eta_{2}, v_{2}\right)\right\|_{X^{s}}(t) \lesssim\left(N_{T}\left(\eta_{1}, v_{1}\right)^{\rho}+\sum_{i=0}^{\rho} N_{T}\left(\eta_{1}, v_{1}\right)^{\rho-i} N_{T}\left(\eta_{2}, v_{2}\right)^{i}\right) \int_{0}^{t} & \frac{d \tau}{(1+\tau)^{\theta \rho}} \\
& \times N_{T}\left(\eta_{1}-\eta_{2}, v_{1}-v_{2}\right) .
\end{aligned}
$$

However, according the inequalities (4.4),

$$
\int_{0}^{t} \frac{d \tau}{(1+\tau)^{\theta \rho}} \lesssim 1
$$




\section{Lemma 4.3}

There exists a constant $C_{s}>0$ such that for all $\left(\eta_{1}, v_{1}\right)$ and $\left(\eta_{2}, v_{2}\right)$ in $X^{s}(\mathbb{R})$, we have

$$
N_{T}\left(\Phi\left(\eta_{1}, v_{1}\right)-\Phi\left(\eta_{2}, v_{2}\right)\right) \leq C_{s}\left(N_{T}\left(\eta_{1}, v_{1}\right)^{\rho}+\sum_{i=0}^{\rho} N_{T}\left(\eta_{1}, v_{1}\right)^{\rho-i} N_{T}\left(\eta_{2}, v_{2}\right)^{i}\right) N_{T}\left(\eta_{1}-\eta_{2}, v_{1}-v_{2}\right) .
$$

and

$$
N_{T}\left(\Phi\left(\eta_{1}, v_{1}\right)\right) \leq C_{s}\left(\left\|\left(\eta_{0}, v_{0}\right)\right\|_{X^{s}}+N_{T}\left(\eta_{1}, v_{1}\right)^{\rho+1}\right) .
$$

Proof. The inequalities (4.9) and (4.10) provide (4.11). For the second inequality, it is enough to take $\left(\eta_{2}, v_{2}\right)=(0,0)$.

Let $M>0$, we consider the closed ball

$$
\bar{B}_{T, M}:=\left\{(\eta, v) \in \mathcal{C}\left([-T, T] ; X^{s}(\mathbb{R})\right) ; N_{T}(\eta, v) \leq M\right\} .
$$

We show that there exists a unique solution $(\eta, v)$ of the system (4.1)-(4.2)-(4.3) in this ball by using the fixed point theorem.

First, there exists $\varepsilon>0$ sufficiently small such that if $\left\|\left(\eta_{0}, v_{0}\right)\right\|_{X^{s}} \leq \varepsilon$, even if we take $C_{s} M$ instead of $M$, it is enough to take $M>0$ satisfying $\varepsilon+M^{\rho+1} \leq M$ so that the inequality (4.12) implies $\Phi\left(\bar{B}_{T, M}\right) \subseteq \bar{B}_{T, M}$. Here, the crucial point is that $\varepsilon$ is independent of $T$. Secondly, we prove that the map $\Phi$ is a contraction on this ball for $M$ sufficiently small. Let $\left(\eta_{1}, v_{1}\right)$ and $\left(\eta_{2}, v_{2}\right)$ be in the closed ball $\bar{B}_{T, M}$. The inequality (4.11) gives

$$
N_{T}\left(\Phi\left(\eta_{1}, v_{1}\right)-\Phi\left(\eta_{2}, v_{2}\right)\right) \leq C_{s} M^{\rho} N_{T}\left(\eta_{1}-\eta_{2}, v_{1}-v_{2}\right),
$$

and, if we need to take $M>0$ smaller again, it gets $C_{s} M^{\rho}<1$. Then, the fixed point theorem is applied and there exists a unique solution of the system (4.1)-(4.2)-(4.3) in the closed ball $\bar{B}_{T, M}$.

It remains to prove that this unique solution can go on in time with all $[0,+\infty[$. By uniqueness of the solution, the inequality (4.12) is written

$$
N_{T}(\eta, v) \leq C_{s}\left(\left\|\left(\eta_{0}, v_{0}\right)\right\|_{X^{s}}+N_{T}(\eta, v)^{\rho+1}\right) .
$$

Since there exists $\varepsilon>0$ sufficiently small such that, if $\left\|\left(\eta_{0}, v_{0}\right)\right\|_{X^{s}} \leq \varepsilon$, we can find $M>0$ such that

$$
\begin{aligned}
N_{0}(\eta, v) & <M \\
C_{s}\left(\varepsilon+M^{\rho+1}\right) & \leq M .
\end{aligned}
$$

Then for all $T>0$, we have $N_{T}(\eta, v)<M$. Indeed, if not by continuity, there exists a time $T>0$ such that

$$
\begin{aligned}
N_{T}(\eta, v) & =M \\
& >C_{s}\left(\varepsilon+M^{\rho+1}\right) \\
& >C_{s}\left(\varepsilon+N_{T}(\eta, v)^{\rho+1}\right),
\end{aligned}
$$

what contradicts the inequality (4.13). To summarize, there exists a constant $M>0$ such that for all $T>0, N_{T}(\eta, v)<M$. In particular, we have for all time $t \geq 0$ and for all $x \in \mathbb{R}$

$$
\begin{aligned}
|\eta(x, t)|+|v(x, t)| & \leq C_{s}\left(\left\|\left(\eta_{0}, v_{0}\right)\right\|_{X^{s}}+N_{T}\left(\eta_{1}, v_{1}\right)^{\rho+1}\right)(1+t)^{-\theta} \\
& \leq C_{s}\left(\varepsilon+M^{\rho+1}\right)(1+t)^{-\theta} .
\end{aligned}
$$

Negative times are dealt with similarly. 


\section{References}

[1] J. Albert, Dispersion of low-energy waves for the generalized Benjamin-Bona-Mahony equation, J. Differential Equations 63 (1986), 117-134.

[2] T. B. Benjamin, J. L. Bona, and J. J. Mahony, Model equations for long waves in nonlinear dispersive systems, Philos. Trans. Roy. Soc. London Ser. A 272 (1972), no. 1220, 47-78.

[3] J.L. Bona, M. Chen, J.-C. Saut, Boussinesq equations and other systems for small-amplitude long waves in nonlinear dispersive media. I. Derivation and linear theory. J. Nonlinear Sci. 12 (2002), no. 4, 283-318.

[4] J.L. Bona, M. Chen, J.-C. Saut, Boussinesq equations and other systems for small-amplitude long waves in nonlinear dispersive media. II. The nonlinear theory. Nonlinearity 17 (2004), no. 3, 925-952.

[5] J. V. Boussinesq, Théorie générale des mouvements qui sont propagés dans un canal rectangulaire horizontal, C.R. Acad. Sci. Paris 73 (1871) 256-260.

[6] T. Kato and G. Ponce, Commutator estimates and the Euler and Navier-Stokes equations, Commun. Pure Appl. Math. 41 (1988), no. 7, 891-907.

[7] D. J. Korteweg and G. de Vries, On the change of form of long waves advancing in a rectangular canal and on a new type of long stationnary waves, Phil. Maj. 39 (1895), 422-443.

[8] Y. Mammeri, On the decay in time of solutions of some generalized regularized long waves equations, Commun. Pure Appl. Anal. 7 (2008), 513-532.

[9] E. Stein, Harmonic Analysis : real-variable methods, orthogonality, and oscillatory integrals, Princeton University Press, 1993.

[10] W. A. Strauss, Dispersion of low-energy waves for two conservative equations, Arch. Rational Mech. Anal. 55 (1974), 86-92.

[11] G. B. Whitham, Linear and Nonlinear Waves, Wiley, New York (1999). 